\title{
Tools for calculations in color space
}

\section{Malin Sjodahl*}

Dept. of Astronomy and Theoretical Physics, Lund University, Sölvegatan 14A, 22362 Lund,

Sweden, E-mail: malin.sjodahlethep.lu.se

Both the higher energy and the initial state colored partons contribute to making exact calculations in QCD color space more important at the LHC than at its predecessors. This is applicable whether the method of assessing QCD is fixed order calculation, resummation, or parton showers. In this talk I will discuss tools for tackling the problem of performing exact color summed calculations. I will start with "theoretical tools" in the form of the (standard) trace bases and the orthogonal multiplet bases (for which a general method of construction was recently presented). Following this, I will focus on two new packages for performing color structure calculations: one easy to use Mathematica package, ColorMath, and one $\mathrm{C}++$ package, ColorFull, which is suitable for more demanding calculations, and for interfacing with event generators.

The European Physical Society Conference on High Energy Physics -EPS-HEP2013

18-24 July 2013

Stockholm, Sweden

${ }^{*}$ Speaker. 


\section{Introduction}

The high energy available at the LHC and the initial state colored partons both contribute to increase the number of colored partons involved in collisions, and indeed, QCD is a major background for most interesting processes. An accurate treatment of QCD and the color space associated with it is therefore of increased importance. This is applicable independent of the method of accessing QCD, fixed order calculations, resummation, or - as lately for the speaker - parton showers [1].

We here discuss both conceptual and theoretical "tools" for dealing with exact calculations in QCD color space. After a few words of introduction in Section 2, we briefly review the standard "trace bases" method in Section 3. In Section 4, we present recent results on how to deal with color space using orthogonal group theory based multiplet bases [2]. Finally, in Section 5, we introduce two computer algebra codes for dealing with calculations in color space, the Mathematica package ColorMath [3], which is designed do deal with color summed calculations of moderate complexity in a user friendly way, and the C++ package ColorFull [4] which aims at dealing with trace bases in an event generator context.

\section{Color space}

Due to confinement we never observe the color of individual partons in QCD. This distinguishes color from other internal degrees of freedom like spin, insofar as for the latter we actually care about in which state a physical object is, rather than just under what representation it transforms. This property also opens up for the possibility to study summed (averaged) quantities only.

It is not hard to argue that for given external partons, the color summed (averaged) space is a finite dimensional vector space equipped with a scalar product

$$
\langle A, B\rangle=\sum_{a, b, c, \ldots}\left(A_{a, b, c, \ldots}\right)^{*} B_{a, b, c, \ldots},
$$

where the sum runs over all quarks, anti-quarks and gluons. For example, if

$$
A=\sum_{g}\left(t^{g}\right)^{a}{ }_{b}\left(t^{g}\right)^{c}{ }_{d}=\sum_{g}^{a}{ }_{b} \longrightarrow 000<{ }_{d}^{c},
$$

then

$$
\langle A \mid A\rangle=\sum_{a, b, c, d, g, h}\left(t^{g}\right)^{b}{ }_{a}\left(t^{g}\right)^{d}{ }_{c}\left(t^{h}\right)^{a}{ }_{b}\left(t^{h}\right)^{c}{ }_{d} .
$$

One way of dealing with the color space is to square the amplitudes one by one as they are encountered. Alternatively - and this is likely the preferred method for processes with many colored partons - one may want to use a basis or a spanning set.

\section{Trace bases}

A standard way of dealing exactly with QCD color space is to note that every four gluon vertex can be rewritten in terms of triple gluon vertices. The triple gluon vertices in turn can be 
replaced using if $f^{a b c}=\left(1 / T_{R}\right)\left[\operatorname{tr}\left(t^{a} t^{b} t^{c}-t^{b} t^{a} t^{c}\right)\right]$, where $T_{R}$ is defined by $\operatorname{tr}\left[t^{a} t^{b}\right]=T_{R} \delta^{a b}$, and all internal gluon propagators can be removed using the Fierz or completeness relation, $\left(t^{a}\right)^{i}{ }_{j}\left(t^{a}\right)^{k}{ }_{l}=$ $T_{R}\left(\delta^{i}{ }_{l} \delta^{k}{ }_{j}-\delta^{i}{ }_{j} \delta^{k}{ }_{l} / N_{c}\right)$.

This can be applied to any QCD amplitude, tree level or beyond, and the result is in general a linear combination of products of traces over gluon indices and traces that have been cut open, i.e. color structures of the form

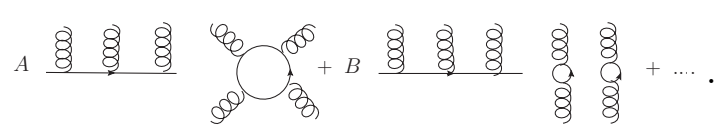

For obvious reasons this type of basis is here referred to as a trace basis.

These bases $[5,6,7,8,9,10,11,12]$ have several advantages. It is easy to see that a basis vector of this type, results in at most two new basis vectors (in a larger vector space) once a gluon is emitted. Furthermore, starting with any basis vector and exchanging a gluon between two partons results in a linear combination of at most four basis vectors [12]. On top of this, powerful recursion relations exist for the amplitudes multiplying the various color structures.

Trace bases, however, also come with significant drawbacks. Most importantly, they are not orthogonal, and for more than $N_{c}$ gluons plus $q \bar{q}$-pairs the "bases" are also overcomplete. As the number of spanning vectors in these bases grows roughly as a factorial in $N_{g}+N_{q \bar{q}}$ this overcompleteness rapidly becomes an issue [2].

\section{Multiplet bases}

It is therefore desirable to use minimal orthogonal bases. As QCD is based on SU(3), one way to construct orthogonal bases is to use bases corresponding to irreducible representations in color space. Basis vectors where at least one subset of partons transforms under a different representation will then automatically be orthogonal. One way to enforce this is to sub-group the partons such that parton 1 and 2 are in a manifest multiplet $M^{12}$, at the same time as partons 1,2 , and 3 are in a manifest multiplet $M^{123}$, etc. [13,2].

As the decomposition of color space into irreducible representations can be enumerated using Young tableau multiplication, the expectations on multiplet type bases are clear: In general (to arbitrary order in perturbation theory), we expect to encounter any state where the incoming partons are in multiplet $M$, and the outgoing partons in the same multiplet $M$. For example, for $q q \rightarrow q q$ we have the Young tableau decomposition

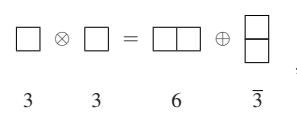

and the corresponding orthogonal basis vectors are

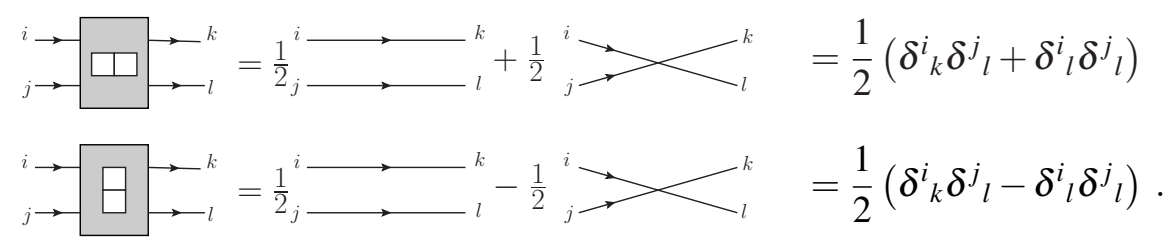


For processes with only quarks and anti-quarks (an incoming anti-quark can always be traded for an outgoing quark etc., so we may always treat the color space as if we had $N_{q}$ incoming quarks and $N_{q}$ outgoing quarks) orthogonal bases can be constructed similarly by using Hermitian versions of Young projection operators $[2,8,14]$.

For processes with gluons, the translation from Young tableaux to basis vectors is far from obvious as the Young tableaux operate with quark units, rather than gluon units. We can enumerate basis vectors using Young tableau multiplication, the problem lays in the construction of the corresponding basis vectors.

Let us start with considering processes with gluons only and consider $g g \rightarrow g g$. Using standard Young tableau multiplication it is easy to find $8 \otimes 8=1 \oplus 8 \oplus 8 \oplus 10 \oplus \overline{10}+\oplus 27 \oplus 0{ }^{1}$. As color is conserved an incoming multiplet of a certain kind can only go to an outgoing multiplet of the same kind, i.e., $1 \rightarrow 1,8 \rightarrow 8 \ldots$, we thus know what basis vectors to expect ${ }^{2}$.

In the case of $g g \rightarrow g g$ this construction problem was solved for $N_{c}=3$ in the 60's [15], and has later been dealt with for general $N_{c}[16,17,18,19]$. For five gluons a multiplet basis was presented in [13]. However, the issue of constructing projection operators for $3 g \rightarrow 3 g$ or more gluons was not addressed until about one year ago when me and Stefan Keppeler presented a general algorithm, applicable to any number of gluons and any $N_{c}$ [2].

Using these projectors, one may also construct orthogonal minimal bases for any number of gluons. As a proof of concept, we have explicitly constructed all (up to) 51 projectors for $3 g \rightarrow 3 g$ (and arbitrary $N_{c}$ ) and all (up to) 265 bases vectors (for arbitrary $N_{c}$ ). The generalization to processes involving both quarks and gluons is straightforward, as each $q \bar{q}$-pair either is in an octet, in which case it can be treated as a gluon, or in a singlet.

These multiplet bases have the advantage of being orthogonal and minimal; for given $N_{c}$ it is easy to cross out basis vectors which are only present for higher $N_{c}$. For few partons this is not important, as only few basis vectors vanish, but for many partons this becomes a significant factor. For example, for 10 gluons there are more than a million basis vectors for general $N_{c}$, but only about hundred thousand for $N_{c}=3$.

Due to the orthogonality of the multiplet bases, these bases may have the potential to very significantly speed up exact calculations in QCD color space.

\section{Computer tools}

In order to facilitate automatic color summed calculations two independent computer algebra codes have been developed and cross checked.

\subsection{ColorFull}

For the purpose of treating a general QCD color structure using trace bases, a C++ color algebra code, ColorFull [4], which creates trace bases for any number and kind of partons and

\footnotetext{
${ }^{1}$ For $N_{c}>3$ there is an additional multiplet which vanishes for $N_{c} \leq 3$.

${ }^{2}$ For reasons to do with the realness of the adjoint representation, the $2 g \rightarrow 2 g$ projection operators actually span the physically relevant four gluon color space. However, in general, this is not the case, i.e., there are basis vectors on the $2 n_{g}$ gluon space, which are not projectors on the $n_{g}$ space.
} 
to any order in $\alpha_{s}$ has been written. ColorFull also describes the effect of gluon emission and exchange, squares color amplitudes and calculates the color soft anomalous dimension matrices.

A separate publication of ColorFull is planned later this year, and pre-release version will soon be publicly available at http://colorfull. hepforge.org/.

\subsection{ColorMath}

ColorMath [3] is a user friendly Mathematica package for calculations in color space of moderate complexity. In its simplest form, the idea of ColorMath is that one should just write down the color structure, much like on paper, and then run CSimplify to contract color indices, for example

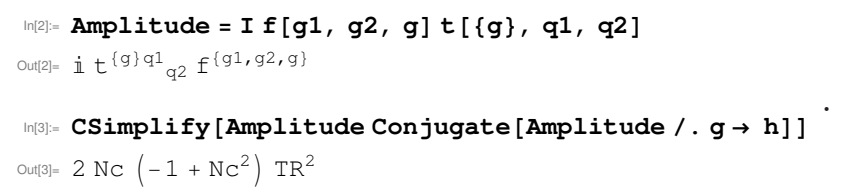

\section{Conclusions}

One way to deal with exact calculations in color space is to use trace bases. This method has advantages when it comes to simplicity, recursion relations and the effect of gluon exchange and gluon emission. It is also the basis for the $\mathrm{C}++$ code ColorFull [4], which is intended for enabling advanced color calculations in an event generators context. This type of basis is, however, overcomplete and not orthogonal, which becomes an obstacle for processes with many partons due to the rapid growth of the number of spanning vectors.

It is therefore desirable to construct minimal orthogonal bases, and in collaboration with Stefan Keppeler I have recently outlined a general recipe for group theory based minimal orthogonal multiplet bases for any QCD process [2]. This can potentially speed up exact calculations in the color space of $\mathrm{SU}\left(N_{c}\right)$ very significantly. In this talk I have also presented a Mathematica package ColorMath [3] for easily performing color summed calculations in $\mathrm{SU}\left(N_{c}\right)$.

\section{Acknowledgments}

Stefan Keppeler is thanked for fruitful collaboration on multiplet bases. A very similar talk was presented at the DIS 2013 conference.

\section{References}

[1] S. Platzer and M. Sjodahl, Subleading $N_{c}$ improved parton showers, JHEP 1207 (2012) 042, [arXiv:1201.0260].

[2] S. Keppeler and M. Sjodahl, Orthogonal multiplet bases in SU(Nc) color space, JHEP 1209 (2012) 124, [arXiv:1207.0609].

[3] M. Sjodahl, ColorMath - A package for color summed calculations in SU(Nc), Eur. Phys. J. C73 (2013) 2310, [arXiv:1211.2099].

[4] M. Sjodahl, ColorFull - A C++ package for color space calculations, work in preparation. 
[5] J. E. Paton and H.-M. Chan, Generalized Veneziano model with isospin, Nucl. Phys. B 10 (1969) $516-520$.

[6] P. Dittner, Invariant tensors in SU(3). II, Commun. Math. Phys. 27 (1972) 44-52.

[7] P. Cvitanović, Group theory for Feynman diagrams in non-Abelian gauge theories, Phys. Rev. D 14 (1976) 1536-1553.

[8] P. Cvitanović, P. Lauwers, and P. Scharbach, Gauge invariance structure of quantum chromodynamics, Nucl. Phys. B 186 (1981) 165-186.

[9] M. L. Mangano, S. J. Parke, and Z. Xu, Duality and multi-gluon scattering, Nucl. Phys. B 298 (1988) 653.

[10] M. L. Mangano, The color structure of gluon emission, Nucl. Phys. B 309 (1988) 461.

[11] Z. Nagy and D. E. Soper, Parton showers with quantum interference, JHEP 09 (2007) 114, [arXiv:0706.0017].

[12] M. Sjodahl, Color structure for soft gluon resummation - a general recipe, JHEP 0909 (2009) 087, [arXiv:0906.1121].

[13] M. Sjodahl, Color evolution of $2 \rightarrow 3$ processes, JHEP 12 (2008) 083, [arXiv: 0807.0555$].$

[14] S. Keppeler and M. Sjodahl, Hermitian Young Operators, arXiv:1307.6147.

[15] A. Macfarlane, A. Sudbery, and P. Weisz, On Gell-Mann's $\lambda$-matrices, $d$ - and f-tensors, octets, and parametrizations of SU(3), Commun. Math. Phys. 11 (1968) 77-90.

[16] P. Butera, G. M. Cicuta, and M. Enriotti, Group weight and vanishing graphs, Phys. Rev. D21 (1980) 972.

[17] P. Cvitanović, Group Theory - Classics Illustrated - part I. Nordita, Copenhagen, 1984.

[18] P. Cvitanović, Group Theory: Birdtracks, Lie’s, and Exceptional Groups. Princeton University Press, 2008. URL: www. birdtracks.eu.

[19] Y. L. Dokshitzer and G. Marchesini, Soft gluons at large angles in hadron collisions, JHEP 01 (2006) 007, [hep-ph/0509078]. 